\title{
Elementos intervinientes de la gestión logística en empresas petroquímicas. Un caso venezolano
}

Elements involved in logistics management in petrochemical companies. A venezuelan case

\section{Carlos Davalillo}

cdavalillo1@gmail.com

ORCID: 0000-0001-9381-0405

Instituto Politécnico Santiago Mariño, Universidad del Zulia -Venezuela
Palabras clave

RESUMEN

(1)

El objetivo de la investigación fue describir los elementos intervinientes de la gestión logística en empresas petroquímicas venezolanas, el caso corresponde específicamente aquellas ubicadas en la Costa Oriental del Lago de Maracaibo. El estudio fue descriptivo, de campo, no experimental. La población estuvo conformada por 15 sujetos. Se aplicó un cuestionario con escala de frecuencias; se empleó el juicio de expertos. Se estimó la confiabilidad por el Coeficiente Alfa de Cronbach, obteniendo 0,987 indicando muy alta confiabilidad. Se aplicó la estadística descriptiva, a través de la media aritmética. Finalmente se concluyó que los elementos intervinientes de la gestión logística en las empresas petroquímicas de la Costa Oriental del Lago poseen una muy alta presencia en términos generales, discriminando los indicadores servicio al cliente, distribución, compras, sistema de información, alcanzaron una muy alta presencia a excepción de los indicadores gestión de inventario y almacenamiento con un resultado de alta presencia.

Distribución, elementos de la gestión logística, gestión logística, gestión de inventario, sistema de información

ABSTRACT The objective of the investigation was to describe the elements involved in logistics management in Venezuelan petrochemical companies, the case specifically corresponds to those located on the eastern shore of Lake Maracaibo. The study was descriptive, field, non-experimental. The population was made up of 15 subjects. A questionnaire with frequency scale was applied; expert judgment was used. Reliability was estimated by Cronbach's Alpha Coefficient, obtaining 0.987 indicating very high reliability. Descriptive statistics were applied through the arithmetic mean. Finally, it was concluded that the elements involved in logistics management in the petrochemical companies of the Eastern Coast of the Lake have a very high presence in general terms, discriminating the indicators of customer service, distribution, purchasing, and information system, reaching a very high presence except for the inventory management and storage, indicator with a high presence result.

Keywords

Distribution, elements of logistics management, logistics management, inventory management, information system 
INTRODUCCIÓN

En el ámbito mundial, toda organización prestadora de servicio, generalmente cuenta con un departamento de servicios logísticos, el cual tiene la responsabilidad de hacer realidad la satisfacción del cliente, la aplicación en la calidad a través del mejoramiento continuo de cada uno de los procesos involucrados, la actualización tecnológica, la responsabilidad profesional, la ética frente a los clientes, el desarrollo de recursos humanos como eje central indispensable e importante.

Tomando en cuenta las afirmaciones de Ballou (2014), explana la gestión logística como aquellas actividades relacionadas con el traslado o almacenamiento de productos que tienen lugar entre los puntos de adquisición y los puntos de consumo. Esta definición implica que la logística empresarial o administración de la cadena de suministros, debe contemplarse como un grupo de actividades relacionadas entre sí, que es necesario administrar coordinadamente.

Dentro de esta perspectiva, y según Salas (2017), el diseño de un sistema de gestión logística en una empresa, comprende la implementación de los procesos de planificación, aprovisionamiento, producción, distribución y servicio al cliente. Sin embargo, para lograr integrar todos estos procesos se hace necesario trabajar en la solidez de los flujos, es decir, decidir sobre la definición de sus redes de distribución, la ubicación de sus almacenes, el modo de gestionar su inventario y el cómo unir todas estas partes con los actores de la cadena de abastecimiento (proveedores, distribuidores y clientes).

En este sentido, las organizaciones prestadoras de servicio se han visto en la necesidad de adaptarse a nuevos cambios operacionales (tecnologías/automatización) cada vez más exigentes, requiriendo para ello, la puesta en marcha de nuevos conceptos y políticas con la finalidad de mejorar el desempeño y el equilibrio, tanto del recurso humano como en el servicio ofrecido al cliente, los cuales se logran mediante la aplicación de nuevos procedimientos y tecnologías que permitan a las empresas optimizar cada uno de sus procesos mediante el análisis de los sistemas de gestión que en ellas son ejecutados.

Si bien es cierto, desde sus inicios y hasta la actualidad, las empresas petroquímicas de Venezuela, siempre realizan grandes esfuerzos por mejorar y modificar sus procesos productivos mediante la aplicación de nuevos estándares de calidad, los cuales proporcionen elementos y aspectos resaltante que conlleven a la satisfacción del cliente.

Ahora bien, en el caso específico de las empresas petroquímicas del Estado Zulia Costa Oriental del Lago entre ellas, el complejo petroquímico Ana María Campo (El Tablazo); Pralca y Oxiteno C.A., las cuales tienen la visión de ser líder en motorizar el desarrollo agrícola e industrial del país, además, de ser reconocida en los mercados nacionales e internacional por la manufactura de productos químicos $y$ petroquímicos de alta calidad y a costos competitivos, capaz de trasformar a Venezuela en potencia petroquímica mundial, para impulsar su desarrollo, mediante la ejecución y aplicación de nuevos procesos estandarizados.

Generalmente, las operaciones de las empresas petroquímicas del Estado Zulia Costa Oriental del Lago están integradas y, por su alta 
productividad, garantizan la eficacia de desempeño de sus productos, los cuales incluyen intermediarios orgánicos, solventes, tensoactivos, fertilizantes, productos químicos industriales, olefinas, resinas plásticas y especialidades químicas, además de producir óxido de etileno y etilenglicol, con la intención de suplir las necesidades de la industria venezolana y destinar parte de la producción a la exportación.

Según entrevistas no estructuradas realizadas al personal, en la actualidad las gerencias de servicios logísticos en las empresas petroquímicas, no cuentan con un sistema de gestión de logística, ni la debida documentación de los procesos que permitan a las organizaciones planificar $y$ coordinar cada una de las operaciones que conforman la cadena logística del transporte terrestre, es decir, no existen las condiciones adecuadas para que las gestiones de los servicios logísticos de transporte terrestre se conviertan en un soporte que asegure la entrega del producto desde el punto de origen hasta su destino final.

Del mismo modo, el personal entrevistado acotó que parte de la flota de transporte (camiones de carga pesada, gandolas con plataformas, camiones cisternas e isotanques) utilizados en las mencionadas empresas son obsoletos, poco eficiente, con costos elevados. Además de ello, no se lleva un control de mantenimiento de los mismos, mediante el cual los tiempos improductivos originados por la falta de mantenimiento del transporte como por la descoordinación en la entrega de los productos al cliente.

Por lo tanto, todo esto conlleva a las empresas a buscar un mecanismo que facilite el análisis, revisión y organización de la documentación, así como acciones estratégicas requeridas para orientar la gestión logística de transporte terrestre en las empresas petroquímicas de la Costa Oriental del Lago.

En este sentido cabe destacar que la verdadera fortaleza de la gestión logística reside en su capital humano, pues son ellos quienes realizan sus funciones en base a sus experiencias como de sus criterios. Sin embargo, al no contar con un instructivo formal y específico que les permita instruirse, establecer, planificar, organizar, reglamentar y sincronizar cada una de las actividades a seguir a fin de determinar las responsabilidades y acciones necesarias para orientar de manera eficiente la gestión logística de transporte terrestre de las empresas petroquímicas de la Costa Oriental del Lago corren el riesgo de no cumplir con los objetivos propuestos en su planificación.

Es importante resaltar, que el transporte terrestre representa uno de los elementos principales en el diseño y administración de un sistema integrado de gestión logística, por cuanto este permite el desplazamiento de personas, materias primas y productos terminados, a través de redes de transporte diseñadas para cumplir despachos en tiempo y forma, al menor costo posible, tanto a nivel local, nacional e internacional (Cruz, 2018).

De los aspectos anteriores surge la inquietud de describir los elementos intervinientes de la gestión logística en empresas petroquímicas venezolanas, específicamente las ubicadas en la Costa Oriental del Lago de Maracaibo. 


\section{Aproximaciones teóricas}

\section{Elementos de la gestión logística}

Una buena logística según Mora (2011), es un elemento clave para la productividad empresarial, al garantizar la satisfacción del cliente. Para ello, se debe prestar especial cuidado a los procesos logísticos de la empresa buscando la manera de optimizarlos para hacerlos más eficientes mediante el buen desempeño de los elementos que la conforman, estos son: servicio al cliente, planificación, gestión de inventario, almacenamiento, distribución, compra, así como poseer un excelente un sistema de información que permita mantener el control y supervisión de lo que sale y entra.

La gestión logística en su forma más primitiva, está compuesta por cinco (5) elementos básicos: planificación logística, servicio al cliente, inventario, almacenamiento y transporte o distribución, según López (2005). De acuerdo a Gambino (2006), la cadena logística está compuesta por cinco (5) elementos sobre los que se trabaja en cualquier gestión: planificación logística, servicio al cliente, manejo de inventario, almacén y transporte. Como puede notarse, ambos autores, coinciden en las posiciones de los elementos que conforman la gestión logística, formando parte del proceso logístico.

En esta perspectiva, para Ballou (2014), los elementos que sirve de apoyo a la logística se clasifican en: planeación, almacenamiento, manejo de materiales (inventarios) y compras. Estos se organizan dependiendo del punto donde pueda tener lugar el canal de suministro de la empresa. Mientras que, según López (2005), la gestión logística es su proceso operativo se caracteriza por la presencia de cinco (5) elementos: planificación, transporte o distribución, inventario, almacenamiento y servicio al cliente.

Para efectos de la investigación, en acuerdo con los postulados planteados por los diferentes autores, la gestión logística en sus procesos operativos se aborda a través de los indicadores: servicio al cliente, planificación, gestión de inventario, almacenamiento, distribución, compras, sistema de información.

\section{Servicio al cliente}

Los estándares de servicio al cliente fijan el nivel de rendimiento y el grado de rapidez al cual debe responder el sistema de logística, al tiempo que afecta los costos que apoyan ese nivel de servicio. Fijar requerimientos de servicios muy altos pueden forzar los costos de logística hasta legar hasta niveles elevados (Anaya, 2014). Por ello los entandares de servicio deben cooperar con el marketing para lograr determinar las necesidades y requerimientos de cliente para la logística del servicio, además de determinar la respuesta del cliente al servicio y fijar niveles de servicio al cliente.

En este marco de referencia, para Casanova y Cuatrecasas (2012), es la diferenciación por canales: mayoristas-minorista-clientes, o por grandes superficies, rapidez, fiabilidad de entrega y sistema de información extremos. Al respecto, Carranza y Sanabria (2005), afirman que el servicio al cliente es el resultado del sistema logístico y la mercadotecnia. El concepto implica la entrega de los productos al consumidor correspondiente en el lugar preciso, en condiciones adecuadas, el tiempo indicado y al menor costo total posible. Todo lo cual puede ser 
coordinado, supervisado e implantado tanto por las funciones logísticas como por los de mercadotecnia.

Según Serna (2006), el servicio al cliente es el conjunto de estrategias que una empresa diseña para satisfacer, mejor que sus competidores, las necesidades y expectativas de sus clientes externos, acatando las políticas, visiones $\mathrm{y}$ misiones ya previamente establecidas por esta entidad siempre en pro del bienestar de la empresa y sus servidores.

Por otro lado, Calderón (2002) destaca que el servicio al cliente se ha denominado como un proceso de satisfacción total, el cual puede describirse como: El proceso integral de cumplir con el pedido de un cliente. Este proceso incluye la recepción del pedido (ya sea manual o electrónica), administración del pago, recolección y empacado de los productos, envío del paquete, entrega del mismo, y manejo de posible devolución de los productos.

De estas definiciones se deduce que el servicio de atención al cliente es indispensable para el desarrollo de una empresa ya que de estas acciones depende la captación de nuevos clientes. Del mismo modo, el éxito de una organización, la reducción de sus costos y la satisfacción de las necesidades de sus clientes, depende de una excelente gestión logística. Así, el nivel de servicio al cliente estará directamente relacionado con la gestión y la efectividad de la cadena: flujo de información, materiales, productos, entre otros. Cuanto más grande sea la organización mayor será la importancia estratégica del servicio al cliente, por lo cual, más importante será colocar la función logística a la par de otras áreas funcionales importantes en termino de rango.

\section{Gestión de inventario}

Para Bowersox y Col. (2007), plantean que al formular una política del inventario, deben considerarse las relaciones específicas de éste. La administración debe comprender estas relaciones para determinar la política del inventario acerca de cuándo hacer e incluso cuánto incluir en un pedido. La política del inventario dirige el desempeño deseado. Los dos indicadores importantes del desempeño del inventario son el nivel de servicio y el no menos importante inventario promedio. Po su parte Cruz (2018) determina que los procesos de la gestión de inventario permiten a la organización decidir los medios a necesitar para realizar sus operaciones y distribuir esos medios en la fabricación del producto deseado, en las cantidades requeridas, en el menor tiempo y costo posible.

Por otra parte, Ballou (2014), considera que los inventarios son acumulaciones de materias primas, provisiones, componentes, trabajo en proceso y productos terminados que aparecen en numerosos puntos a lo largo del canal de producción y de logística de una empresa. Los inventarios se hayan con frecuencia en lugares como almacenes, patios, pisos de las tiendas, equipo de transporte y en los estantes de las tiendas de menudeo.

Para Casanova y Cuatrecasas (2012), la gestión de inventario se refiere a la actividad que tiene como objetivo fundamentado, realizar la gestión para proporcionar la requerida disponibilidad de los bienes que solicita la demanda. Esta misma actividad es llamada por Ballou (2014), manejo de inventario, e implica equilibrar la disponibilidad de producto (o servicio al cliente) con los costos de suministrar un nivel determinado de disponibilidad del 
producto. Siendo el principal objetivo del manejo de inventario asegurar que el producto esté disponible en el momento y en las cantidades deseadas.

En lo expuesto anteriormente según los autores referidos la gestión de inventario es un componente del proceso y parte básica de un sistema de logística, se debe acentuar que los inventarios pueden fallar por diferentes motivos, sobre todo en la actual economía se observan muchas desviaciones en la cadena de suministros, además los inventarios pueden traer consigo altos costos de mantenimientos y de traslados si no se cuentan con una estrategia adecuada en su manejo.

\section{Almacenamiento}

De acuerdo con Pulido (2009), el almacenamiento forma parte de todas aquellas actividades que permiten el correcto almacenaje de productos y la preparación de pedidos. Muchas veces se le entiende simplemente como el almacenamiento en sí, lo cual en una simplificación errónea. De ahí, algunas empresas perciben el almacenamiento como un campo de desarrollo crucial y, en algunos casos, como una competencia central, por lo tanto, el almacenamiento es un arma estratégica usada para mejorar la posición competitiva.

Para Bowersox y col. (2007), al planificar la disposición de un almacén, es esencial que se asignen lugares específicos a los productos, llamados cajones, con base en sus características individuales. Las variables más importantes de los productos para considerar en un plan de asignación de cajones son los requerimientos de velocidad, peso y almacenamiento especial de los productos. La velocidad de un producto es el factor más importante que dirige la disposición del almacén.

Los productos con un volumen alto deben ubicarse de modo que minimicen la distancia de los movimientos. Por ejemplo, los productos con velocidad alta deben colocarse cerca de las puertas, los pasillos principales y los niveles más bajos de los anaqueles de almacenamiento. Dichas posiciones minimizan el manejo y evitan izar los productos. Por otra parte, se suelen asignar posiciones más alejadas de los pasillos principales y en los anaqueles más altos a los productos con un volumen bajo.

Asimismo, el plan de almacenamiento debe tomar en consideración el peso y las características especiales del producto. Debe asignarse un lugar cerca del piso a los artículos relativamente pesados para evitar pisarlos. Los productos voluminosos o de baja densidad requieren espacio por unidad de volumen. El espacio de piso a lo largo de los muros exteriores es ideal para tales artículos. Por otra parte, los artículos más pequeños pueden requerir gabinetes, recipientes o cajones.

López (2014), el almacenamiento es aquel proceso mediante el cual se ubican, acopian e identifican los materiales dentro de las facilidades físicas existentes en los almacenes, siguiendo los procedimientos establecidos por la empresa. Son manejados a través de una política de inventario. Esta función controla físicamente de la misma manera mantiene todos los artículos inventariados. Al elaborar la estrategia de almacenamiento se deben definir de manera coordinada el sistema de gestión del almacén y el modelo de almacenamiento. En este indicador los autores dejan claro que el almacenamiento es un punto esencial dentro de un sistema de 
logística, dependiendo del producto que se comercializa este debe poseer un sistema eficaz capaz de evitar pérdidas y atrasos en las entregas.

Para efectos de la presente investigación, se considera el proceso de almacenamiento, como el conjunto de actividades inherentes a mantener la disponibilidad de todos los recursos necesarios en el momento, lugar y cantidad concreta, sin duda al mínimo costo. Así, el proceso de almacenamiento debe estar asociado con la óptima gestión logística de aquellos recursos que deben ser resguardados hasta tanto estén a disposición de los consumidores, prestación de servicios al cliente, entre otros.

\section{Distribución}

Sánchez y Méndez (2006), establecen que la distribución es la parte de la logística que hace referencia al movimiento externo de los productos desde el vendedor al cliente o comprador. Se considera en adelante que logística y distribución física son términos equivalentes y que abarcan aquel conjunto de operaciones llevadas a cabo para que el producto recorra el camino que dista desde su punto de producción hasta el consumo. Los canales de distribución son los que posibilitan el desarrollo de estas actividades. Un sistema de distribución eficaz es aquel que tenga a disposición del mercado los productos que éste demande, en la cantidad precisa y en momento oportuno, para esto debe disponerse de unos medios logísticos adecuados, que suministren los productos a los canales de distribución.

Para alcanzar una logística eficiente debe llevarse a cabo al menor costo posible, no obstante, al contemplar los costos de la distribución física deben tenerse en cuenta, no solo los costos directos de cada una de las actividades que engloba (procesamiento de pedidos, transportes, almacenaje, entre otros.), sino también la interpelación entre cada una de ellas y los costos de oportunidad en que pueden cubrirse. El cumplimiento de los objetivos de la distribución física debe contemplarse desde una perspectiva global. Es decir, se trata de minimizar el costo total del sistema de distribución física, para el mejor desempeño de las organizaciones.

Anaya (2014) establece que la distribución forma parte los procesos, almacenaje $y$ distribución física propiamente dicho. Comienza con la recepción de productos terminados en el almacén y continua con los procesos de identificación, registro ubicación, custodia y control, cuya finalidad es que el producto esté disponible para la red de ventas.

En contraste Talaya afirma que la distribución es un conjunto de actividades interrelacionadas llevadas a cabo por una organización o por la integración de varias necesarias para gestión eficiente del flujo de producción desde el almacén o la fabricación hasta el usuario, desarrollando para ello un sistema de comunicaciones que posibilite su entrega en el momento oportuno y en la forma y lugar adecuado.

Desde el punto de vista del investigador la distribución se considera como el proceso mediante el cual se recibe la materia prima para elaborar el producto se almacena y a su vez es traslado a los centros de distribución los cuales sirven como medio de entrega para los clientes. 


\section{Compras}

Según Coronado (2008), la compra consiste en obtener productos o servicios de la calidad adecuada, con el precio justo, en el tiempo indicado y en el lugar preciso. Muchas empresas manejan este concepto cómo la acción de adquirir provisiones o materiales para la empresa (materias primas, componentes o artículos terminados. También se puede decir que compras es comerciar, es el acto de obtener el producto o servicio de la calidad correcta, al precio correcto, en el tiempo correcto y en el lugar correcto.

Es necesario comentar también que el proceso de compras no sólo implica el adquirir y obtener los productos que se requieran en una empresa, sino que también incluye el negociar los precios y la calidad de los productos a comprar con la finalidad de traer beneficios a la empresa sobre todo en términos monetarios.

En esta perspectiva Mercado (2006), define la compra como aquella operación que involucra todo el proceso de ubicación de proveedor o fuente de abastecimiento, adquisición de materiales a través de negociaciones de precios y condiciones de pago o el proveedor elegido y la recepción de las mercaderías correspondientes para controlar y garantizar el suministro de la adquisición.

Las compras, según Serna (2006), es una actividad de apoyo al proceso logístico que tiene como objetivo adquirir aquellos bienes o servicio que la empresa necesita, garantizando el abastecimiento de las cantidades requeridas en el momento preciso y en las mejores condiciones posibles de calidad y precio. A través de ella se seleccionan las fuentes, se determinan las cantidades a adquirir y el momento de efectuar las adquisiciones y la planificación de los productos. Impacta en la gestión de inventario.

De lo expuesto, el investigador infiere que las compras, son una actividad de apoyo a la logística involucra la adquisición de materias primas, componentes y suministros para la organización. Las actividades asociadas a esta parte del proceso logístico incluyen desde la detención de la necesidad hasta la especificación de la forma en la que se reciben los bienes o servicios.

\section{Sistema de información}

Considerando a las autoras Martínez y Brenzini (2015), los sistemas de información se relacionan con todas las actividades desarrolladas en las empresas y estas dependen, en su mayoría, aunque de modos diferentes, del sistema. Por tanto, los cambios en dichos sistemas alteran la dinámica de la organización y viceversa. Para las organizaciones, en el entorno actual, el cambio se ha convertido en una constante. Los sistemas de información pueden constituir verdaderos instrumentos o impedimentos para ese cambio. Por ello, tanto los directivos como los gerentes no deben dejar solamente a los especialistas la importante labor del desarrollo de sistemas de información. Aún más si se tiene en cuenta que influyen y pueden estar influidos por las características propias de la organización a la que pertenecen: cultura organizativa, estructura inicial, procedimientos, entre otros.

Para Fernández (2010), los sistemas de información interactúan entre sí y proporcionan información tanto para las necesidades de las operaciones como de la administración. En teoría, una computadora no es necesariamente un ingrediente de un Sistema de Información 
Gerencial (SIG), pero en la práctica es poco probable que exista un SIG complejo sin las capacidades de procesamiento de las computadoras.

Para Amaya (2009) el sistema de información referido al sector logístico u otros sectores, la información rápida y actualizada de todo lo ocurrido en el entorno es un elemento trascendental en la actividad diaria. Por tal motivo, la información mediante los sistemas empleados debe contar con un respaldo, para garantizar el uso de la misma a futuro, siendo base para la construcción de nuevas estrategias informáticas.

Así mismo, mencionan Herederos y Joaquín (2006), la información constituye uno de los activos estratégicos más importantes para la empresa. El funcionamiento de toda organización depende de un adecuado abastecimiento, tratamiento y en definitiva gestión de ese recurso el cual constituye la información.

Desde otra perspectiva Laudon y Laudon (2012) consideran el sistema de información como un conjunto de información extensa y coordinada de subsistemas racionalmente integrados que transforman los datos en información en una variedad de formas para mejorar la productividad de acuerdo con los estilos y características de los administradores.

Para efectos de la investigación, la información obtenida a tiempo permite decisiones más atinadas y precisas. En este contexto, una buena o mala estrategia de sistemas de información es determinante para el éxito o el fracaso de los negocios de una empresa. El hardware, software y comunicaciones son herramientas imprescindibles para los negocios Incluirlas dentro de un plan de sistemas adecuado es vital para las compañías globalizadas, en la actualidad la mayoría de las empresas dependen de un buen sistema de información ya que de ello depende el éxito de sus procesos en todos sus departamentos como: compras, cuentas por pagar, cobrar entre otros.

\section{MATERIALES Y METODO}

La investigación obedece al tipo descriptiva, ésta tiene como propósito interpretar realidades de hechos, incluye descripción, registro, análisis e interpretación de la naturaleza actual, composición o procesos de los fenómenos (Hernández et al., 2014). Con relación al diseño Arias (2012) expresa que el diseño de campo consiste en la recolección de datos directamente de la realidad donde ocurren los hechos, sin manipular o controlar alguna variable.

De todo lo anteriormente indicado se pretende recopilar directamente la información detallada y exacta específicamente en las organizaciones objeto de investigación, es decir en el Complejo Petroquímico el Tablazo, Pralca y Oxiteno. El diseño de campo utilizado en este estudio se corresponde con el no experimental y transaccional, puesto que no se manipularán las variables, solo se tomarán los datos en un solo momento para su posterior análisis.

En el caso particular de este estudio investigativo, la población es pequeña por lo que se abordó a través de un muestreo censal o censo poblacional abarcando la totalidad de la población. Esta investigación comprende una población finita, constituida por las tres (3) empresas Petroquímicas de la Costa Oriental del Lago, entre ellas: Pralca, Complejo Ana María Campos (El Tablazo) y Oxiteno, únicas organizaciones en la Costa Oriental del Lago 
encargada de la producción de producto químico de uso industrial. Finalmente, la población estuvo representada por quince (15) sujetos informantes entre los cuales se señalan: Gerentes Líderes, supervisores y operadores de transporte (Choferes).

Se utilizó como técnica la encuesta y el instrumento de recolección de datos fue un cuestionario. Con relación a la validez del contenido del instrumento se obtuvo mediante el juicio de cuatro (04) expertos. Se procesaron los datos de manera automatizada utilizando el programa SPSS V.20 el cual permitió calcular el coeficiente, Alpha Cronbach ubicando el rango de efectividad en 0,987, es decir una confiabilidad Muy alta. Con relación al análisis de los datos se realizó mediante la estadística descriptiva, con base en la media aritmética.

A continuación, se presenta el cuadro 1 donde se muestra la categoría de análisis para la interpretación de la media.

Cuadro 1. Baremo para la interpretación de la media aritmética

\begin{tabular}{ccc}
\hline Rango & Alternativa & Categoría \\
\hline $\mathbf{4 . 2 1}-\mathbf{5 . 0 0}$ & Siempre & Muy alta presencia \\
$\mathbf{3 . 4 1}-\mathbf{4 . 2 0}$ & Casi siempre & Alta presencia \\
$\mathbf{2 . 6 1}-\mathbf{3 . 4 0}$ & A veces & Moderada presencia \\
$\mathbf{1 . 8 1}-\mathbf{2 . 6 0}$ & Casi Nunca & Baja presencia \\
$\mathbf{1 . 0 0}-\mathbf{1 . 8 0}$ & Nunca & Muy baja presencia \\
\hline
\end{tabular}

Fuente: Elaboración propia (2019)

\section{RESULTADOS Y DISCUSION}

Con relación a los elementos intervinientes de la gestión logística en empresas petroquímicas se presentan los resultados obtenidos del procesamiento de datos, a partir de los indicadores descritos a continuación:

\section{Dimensión: Elementos de la gestión logística}

\section{Indicador: Servicio al cliente}

Según los datos aportados por la tabla 1 , para el ítem 1, el 33,33\% seleccionó la alternativa "siempre", el 46,67\% optó por la alternativa "casi siempre" y una minoría distribuida en un $6,67 \%$ seleccionaron las alternativas "a veces", "casi nunca" y "nunca"; con relación a la media del ítem se aprecia un 3,39 ostentando la categoría moderada presencia. Por su parte el ítem 2 el 40,00\% seleccionó la alternativa "siempre", el 60,00\% optó por la alternativa "casi siempre"; mientras la media del ítem se aprecia un valor de 4,40 con la categoría de moderada presencia; con relación a la media del indicador servicio al cliente alcanzó una media de 4,17 categorizado como alta presencia.

Es significativo mencionar lo señalado por Anaya (2014), Serna (2006), Casanova y Cuatrecasas (2012), quienes consideran que el servicio al cliente un factor competitivo, y su calidad se traduce en el número de usuarios o compradores en la industria. Lamentablemente, 
las empresas que no toman en serio el servicio al cliente han fracasado, perdiendo con ello clientes, tiempo y dinero, se deben alcanzar entandares de servicio al cooperar con el marketing para lograr determinar las necesidades y requerimientos de cliente. Por ello, el cliente es el activo más valioso que posee una organización, por tanto es necesario que las empresas enfoquen sus esfuerzos en la articulación sistemática y armoniosa de los procesos como las acciones que buscan lograr un cliente satisfecho.

Tabla 1. Resultados obtenidos del indicador: Servicio al cliente

\begin{tabular}{|c|c|c|c|c|}
\hline $\begin{array}{c}\text { Indicador: servicio } \\
\text { al cliente }\end{array}$ & $\begin{array}{l}\text { Ítem } 1 . \\
\text { estrategias } \\
\text { para ser má }\end{array}$ & $\begin{array}{l}\text { diseña } \\
\text { cliente }\end{array}$ & \multirow{2}{*}{\multicolumn{2}{|c|}{$\begin{array}{c}\text { Ítem 2. El servicio al cliente está } \\
\text { enfocado en el alcance de la } \\
\text { satisfacción. }\end{array}$}} \\
\hline Alternativa & \multicolumn{2}{|r|}{ Fr (\%) } & & \\
\hline 1 Siempre & 5 & 33,33 & 6 & 40,00 \\
\hline 2 Casi siempre & 7 & 46,67 & 9 & 60,00 \\
\hline 3. A veces & 1 & 6,67 & 0 & 0 \\
\hline 4. Casi nunca & 1 & 6,67 & 0 & 0 \\
\hline 5. Nunca & 1 & 6,67 & 0 & 0 \\
\hline Total & 15 & 100 & 15 & 100 \\
\hline Media ítem & \multicolumn{2}{|c|}{3,39} & \multicolumn{2}{|c|}{4,40} \\
\hline Categoría & \multicolumn{2}{|c|}{ Moderada presencia } & \multicolumn{2}{|c|}{ Muy alta presencia } \\
\hline Media indicador & \multicolumn{4}{|c|}{4,17} \\
\hline Categoría & \multicolumn{4}{|c|}{ Alta presencia } \\
\hline
\end{tabular}

Fuente: Elaboración propia (2019)

\section{Indicador: Gestión de inventario}

Mediante los datos reflejados por la tabla 2 , según el ítem 3 , se puede notar que una mayoría de los encuestados representados por un 46,67\%, opinan que "siempre" en las empresas a las cuales representan, el servicio al cliente está enfocado en el alcance de la satisfacción, mientras que otro sector importante representado por un 40,00 \% aseguró que "casi siempre", una minoría el $13,33 \%$ respondió "a veces"; alcanzando un valor para la media del ítem de 4,33 con una categoría de muy alta presencia.
Analizando el ítem 4, el 13,33\%, seleccionaron la alternativa "siempre", el representado por un 6,67 \% optaron por las alternativas que "casi siempre" y "a veces"; una mayoría el 73,33 \% respondió "casi nunca"; alcanzando un valor para la media del ítem de 2,60 con una categoría de moderada presencia; con relación a la media del indicador gestión de inventario se obtuvo un valor de 3,47 ostentando una categoría de alta presencia. 
De estos resultados se puede interpretar que no todas las empresas objeto de estudio analizan los costos de aprovisionamiento del stock de inventario. Cabe destacar que esta situación pone a dichas empresas en desventaja según lo considerado por Cruz (2018) y Casanova y Cuatrecasas (2012), estos autores apuntan que los inventarios representan un uso importante del capital en el canal de suministros.

Tabla 2. Resultados obtenidos del indicador: Gestión de inventario

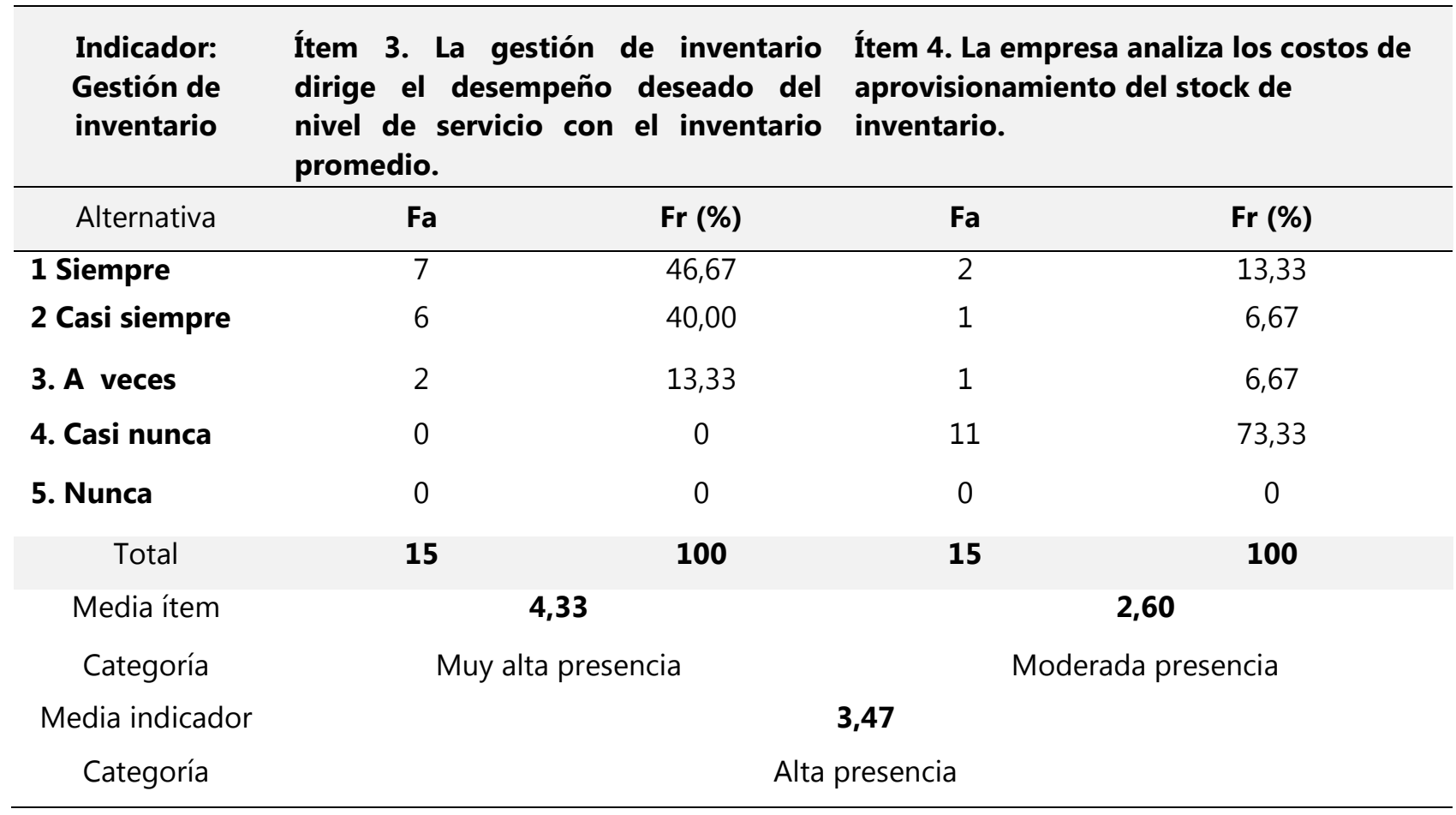

Fuente: Elaboración propia (2019)

Su buena administración supone mantenerlos al nivel más bajo posible, en un equilibrio de costos directos e indirectos, asegurando a su vez un nivel deseado de disponibilidad de producto. Por su parte Ballou (2014), afirma que el inventario equilibra la disponibilidad de producto con los costos a un nivel determinado de disponibilidad del producto, cuyo principal objetivo es asegurar la disponibilidad del producto en el momento $y$ en las cantidades deseadas.

\section{Indicador: Almacenamiento}

De los resultados reflejados en la tabla 3, para el ítem 5 el 26,67\% optó por la alternativa "siempre", una mayoría representada por un $66,67 \%$ seleccionó la alternativa "casi siempre" y una pequeña porción el 6,67\% optó por la alternativa que "a veces"; con una media el ítem de 4,20 categorizada como alta presencia. 
Tabla 3. Resultados obtenidos del indicador: Almacenamiento

\begin{tabular}{|c|c|c|c|c|}
\hline \multirow{2}{*}{$\begin{array}{c}\text { Indicador: } \\
\text { Almacenamiento } \\
\text { Alternativa }\end{array}$} & \multicolumn{2}{|c|}{$\begin{array}{l}\text { Ítem } 5 . \text { El almacenamiento } \\
\text { está manejado a través de la } \\
\text { Política de inventario. }\end{array}$} & \multicolumn{2}{|c|}{$\begin{array}{l}\text { Ítem 6. El almacenamiento de la empresa } \\
\text { permite controlar eficientemente el flujo de } \\
\text { materiales, desde el punto de origen hasta el } \\
\text { punto de consumo. }\end{array}$} \\
\hline & $\mathbf{F a}$ & Fr (\%) & $\mathbf{F a}$ & Fr (\%) \\
\hline 1 Siempre & 4 & 26,67 & 5 & 33,33 \\
\hline 2 Casi siempre & 10 & 66,67 & 8 & 53,33 \\
\hline 3. A veces & 1 & 6,67 & 2 & 13,33 \\
\hline 4. Casi nunca & 0 & 0 & 0 & 0 \\
\hline 5. Nunca & 0 & 0 & 0 & 0 \\
\hline Total & 15 & 100 & 15 & 100 \\
\hline Media ítem & \multicolumn{2}{|c|}{4,20} & \multicolumn{2}{|c|}{4,20} \\
\hline Categoría & \multicolumn{2}{|c|}{ Alta presencia } & \multicolumn{2}{|c|}{ Alta presencia } \\
\hline Media indicador & \multicolumn{4}{|c|}{4,20} \\
\hline Categoría & \multicolumn{4}{|c|}{ Alta presencia } \\
\hline
\end{tabular}

Fuente: Elaboración propia (2019)

En lo que respecta al ítem 6 el 33,33\% optó por la alternativa "siempre", la mayoría representada por un 53,33\% seleccionó la alternativa "casi siempre" y el 6,67\% optó por la alternativa "a veces"; con una media el ítem de 4,20 categorizada como alta presencia: Por su parte la media del indicador también coincidió en 4,20 y la categoría alta presencia.

Con relación a los resultados obtenidos, y considerando los postulados teóricos de Pulido (2009), y Bowersox y col. (2007) y López (2014) afirman que poniendo en práctica un óptimo almacenamiento, este le proporciona a cualquier empresa, ratifican la importancia de planificar la disposición de un almacén, para asignar lugares específicos a los productos, garantizando un eficiente flujo de materiales a través de la política de inventario, a fin de controlar eficientemente el flujo de materiales, desde el punto de origen hasta el punto de consumo.

\section{Indicador: Distribución}

En la tabla 4, se muestran los resultados correspondientes al ítem 7, donde la mayoría de los encuestados representados por un $80,00 \%$ opinaron que "siempre" la actividad de distribución en la empresa, abarca el conjunto de operaciones llevadas a cabo para que el producto recorra el camino que dista desde su punto de producción hasta el consumo. Mientras que un $20 \%$ optó por la opción "casi siempre"; con una media del ítem de 4,80 una muy alta presencia. 
Tabla 4. Resultados obtenidos del indicador: Distribución

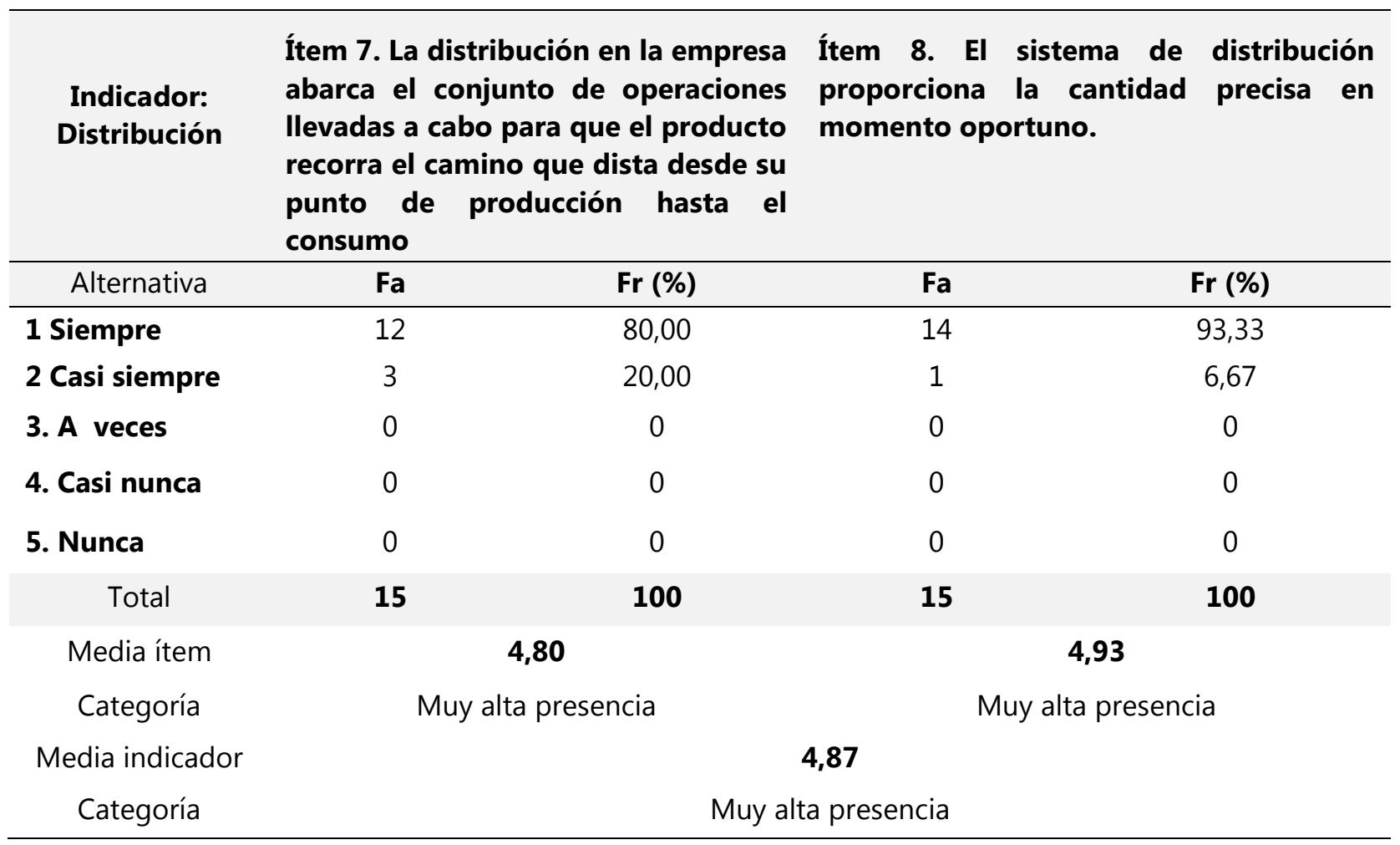

Fuente: Elaboración propia (2019)

Respecto al ítem 8 una gran mayoría de los encuestados representados por un 93,33\% opinaron que "siempre" el sistema de distribución proporciona la cantidad precisa en momento oportuno; mientras que un $6,67 \%$ optó por la opción "casi siempre"; con una media del ítem de 4,93 una muy alta presencia; con relación a la media del indicador se obtuvo un valor de 4,87 categorizado con una muy alta presencia.

Los resultados anteriores coinciden con Sánchez y Méndez (2006), Anaya (2014) y Talaya (2008) quienes establecen que la distribución es la parte de la logística dirigida al movimiento externo de los productos desde el vendedor al cliente o comprador, para esto debe disponerse de unos medios logísticos adecuados, que suministren los productos a los canales de distribución.

\section{Indicador: Compras}

Según los datos aportados por la tabla 4, para el ítem 9, la mayoría de los entrevistados representados en un $86,67 \%$, opinan que la empresa "siempre" realiza la gestión de compras en función de obtener bajos costos con alta calidad de los productos adquiridos, mientras un $13 \%$ seleccionó la alternativa "casi siempre"; obteniéndose una media del ítem de 4,87 categorizado como muy alta presencia.

Con relación al ítem 10, la mayoría de los encuestados representados por un $80 \%$ opinaron que la empresa "siempre" aplica la gestión de compras mediante el análisis de las 
necesidades de la organización, mientras un $13,33 \%$ seleccionó la alternativa "casi siempre" y un $6,67 \%$ "a veces"; obteniendo una media del ítem de 4,73 categorizado como muy alta presencia; se obtuvo una media de 4,80 para el indicador, alcanzando una categoría de muy alta presencia.

Tabla 5. Resultados obtenidos del indicador: Compras

\begin{tabular}{|c|c|c|c|c|}
\hline \multirow{2}{*}{$\begin{array}{c}\text { Indicador: } \\
\text { Compras } \\
\text { Alternativa }\end{array}$} & \multicolumn{2}{|c|}{$\begin{array}{l}\text { Ítem 9. La gestión de compras se } \\
\text { realiza en función de obtener bajos } \\
\text { costos con alta calidad de los } \\
\text { productos adquiridos }\end{array}$} & \multicolumn{2}{|c|}{$\begin{array}{l}\text { Ítem 10. La gestión de compras se } \\
\text { aplica mediante el análisis de las } \\
\text { necesidades de la organización. }\end{array}$} \\
\hline & $\mathbf{F a}$ & Fr (\%) & $\mathbf{F a}$ & $\operatorname{Fr}(\%)$ \\
\hline 1 Siempre & 13 & 86,67 & 12 & 80,00 \\
\hline 2 Casi siempre & 2 & 13,33 & 2 & 13,33 \\
\hline 3. A veces & 0 & 0 & 1 & 6,67 \\
\hline 4. Casi nunca & 0 & 0 & 0 & 0 \\
\hline 5. Nunca & 0 & 0 & 0 & 0 \\
\hline Total & 15 & 100 & 15 & 100 \\
\hline Media ítem & \multicolumn{2}{|c|}{4,87} & \multicolumn{2}{|c|}{4,73} \\
\hline Categoría & \multicolumn{2}{|c|}{ Muy alta presencia } & \multicolumn{2}{|c|}{ Muy alta presencia } \\
\hline Media indicador & \multicolumn{4}{|c|}{4,80} \\
\hline Categoría & \multicolumn{4}{|c|}{ Muy alta presencia } \\
\hline
\end{tabular}

Fuente: Elaboración propia (2019)

Los resultados validan lo señalado por Serra (2005) que toda empresa debe aplicar la gestión de compras mediante el análisis de necesidades considerando: definición de los procesos para la gestión del aprovisionamiento, identificar las necesidades, elegir las fuentes de suministro, manejar la negociación y contratación, seguimiento y revisión de pedidos, control de pago de facturas, entre otros.

Es importante indicar que este pequeño número de empresas no realiza la gestión de compras oportunamente, debería considerar lo planteado por Mercado (2006), quien señala que la práctica de una correcta gestión de compras asegura que la empresa tenga los mejores proveedores para abastecer los mejores productos y servicios, al mejor valor total. La función de compras a menudo gasta más dinero que cualquier otra función de la empresa, así que las compras proporcionan una buena oportunidad para reducir los costos y aumentar los márgenes de beneficio.

\section{Indicador: Sistema de información}

Apreciando los datos aportados por la tabla 5, para el ítem 11 puede observarse como una mayoría de los entrevistados representados por un $93,33 \%$ indican que sus empresas "siempre" 
los sistemas de información de gestión logística les brindan el apoyo; mientras que una minoría constituida por un $6,67 \%$ seleccionó la alternativa "casi siempre"; obteniéndose 4,93 para la media del ítem categorizado como muy alta presencia.

Según los resultados del ítem 12 se observa que el $100 \%$ de los encuestados opinaron que "siempre" los sistemas de información proporcionan información de apoyo a la toma de decisiones, logrando ventajas competitivas a través de su implantación; con un valor de 5,00 para la media del ítem y 4,97 para la media del indicador.

Los resultados anteriores coinciden con Amaya (2009), Fernández (2010), Herederos y Joaquín (2006), Laudon y Laudon (2012), y
Martínez y Brenzini (2015) para quienes los sistemas de información juegan un papel muy importante en el ámbito empresarial, por ser una herramienta poderosa y alterna para apoyar a los gerentes individuales a tomar mejores decisiones. Los sistemas de información son claves cuando los problemas no son los habituales y cambian constantemente permitiendo así tomar decisiones de manera más eficiente. Otra función de los sistemas de información es el apoyo a la alta dirección al monitorear el desempeño de la empresa, identificar problemas estratégicos y oportunidades de los negocios. Esta situación les aporta diversas ventajas a las empresas no solo de mantenerse en el mercado sino de expandir sus sucursales y ofertas de servicios.

Tabla 6. Resultados obtenidos del indicador: Sistema de información

\begin{tabular}{|c|c|c|c|c|}
\hline \multirow{2}{*}{$\begin{array}{c}\begin{array}{c}\text { Indicador: } \\
\text { sistema de } \\
\text { información }\end{array} \\
\text { Alternativa }\end{array}$} & \multicolumn{2}{|c|}{$\begin{array}{l}\text { Ítem 11. El sistema de información de } \\
\text { la gestión logística permite el apoyo } \\
\text { en la toma de decisiones operativas }\end{array}$} & \multicolumn{2}{|c|}{$\begin{array}{l}\text { Ítem 12. Los sistemas de información } \\
\text { proporcionan información de apoyo a la } \\
\text { toma de decisiones, logrando ventajas } \\
\text { competitivas a través de su implantación. }\end{array}$} \\
\hline & $\mathrm{Fa}$ & Fr (\%) & $\mathbf{F a}$ & Fr (\%) \\
\hline 1 Siempre & 14 & 93,33 & 15 & 100 \\
\hline 2 Casi siempre & 1 & 6,67 & 0 & 0 \\
\hline 3. A veces & 0 & 0 & 0 & 0 \\
\hline 4. Casi nunca & 0 & 0 & 0 & 0 \\
\hline 5. Nunca & 0 & 0 & 0 & 0 \\
\hline Total & 15 & 100 & 15 & 100 \\
\hline Media ítem & \multicolumn{2}{|c|}{4,93} & \multicolumn{2}{|c|}{5,00} \\
\hline Categoría & \multicolumn{2}{|c|}{ Muy alta presencia } & \multicolumn{2}{|c|}{ Muy alta presencia } \\
\hline Media indicador & \multicolumn{4}{|c|}{4,97} \\
\hline Categoría & \multicolumn{4}{|c|}{ Muy alta presencia } \\
\hline
\end{tabular}

Fuente: Elaboración propia (2019) 
Entre los beneficios que pueden aportar los sistemas de información como apoyo a la toma de decisiones administrativas para la organización estarían reflejados en la cadena de abastecimiento, relaciones con el cliente y/o usuarios y la planeación de escenarios de negocios.
Una vez analizados e interpretados los elementos intervinientes de la gestión logística, a continuación, se presenta la tabla 6, resumiendo el comportamiento de cada indicador, sirviendo como punto de partida para la discusión de los resultados alcanzados por la misma.

Tabla 7. Elementos de la gestión logística

\begin{tabular}{lcc}
\hline Indicadores & $\overline{\boldsymbol{X}}$ & Categorías \\
\hline Servicio al cliente & 4,17 & Muy alta presencia \\
Gestión de inventario & 3,47 & Alta presencia \\
Almacenamiento & 4,20 & Alta presencia \\
Distribución & 4,87 & Muy alta presencia \\
Compras & 4,80 & Muy alta presencia \\
Sistema de Información & 4,97 & Muy alta presencia \\
& $\mathbf{4 , 4 1}$ & Muy alta presencia \\
\hline
\end{tabular}

Fuente: Elaboración propia (2019)

Finalmente, el comportamiento de los elementos de la gestión logística, se evidencia según las respuestas de los encuestados que posee una muy alta presencia en la gestión logística en las empresas petroquímicas de la Costa Oriental del Lago, al obtener una media aritmética de 4,41 evidenciando una muy alta presencia.

\section{CONCLUSIONES}

La realidad competitiva en las empresas, presenta un campo de batalla en donde la flexibilidad, la velocidad de llegada al mercado y la productividad son las variables claves que determinan su permanencia en los mercados, es aquí donde la logística juega un papel crucial, a partir del manejo eficiente del flujo de bienes y servicios hacia el consumidor final.

La gestión logística implica una nueva forma de integrar, gestionar y controlar los procesos de una empresa. Las estructuras organizacionales van conformando sus modelos sobre la base de sus procesos más que en sus áreas funcionales, esto permite, por un lado el desarrollo de la competitividad empresarial por parte de las empresas, por otro lado, la creación y el mantenimiento de la misma dentro del sistema económico.

Considerando la evolución acelerada de la gestión logística y las oportunidades de una 
apertura comercial cada vez más desarrollada, inciden en el interés por la logística como un aspecto al interior de la organización. Hoy en día es un proceso transversal, por cuanto un error en alguna de las etapas de la cadena de suministro (aprovisionamiento, producción, almacenamiento, transporte $y$ servicio al cliente), puede afectar a múltiples áreas de la empresa y acarrear graves consecuencias para el negocio.

De acuerdo con las consideraciones anteriores, a continuación, se presentan los aspectos más significativos con relación a los elementos de la gestión logística a saber: servicio al cliente, gestión de inventario, almacenamiento, distribución, compras y sistema de información; se evidenció que en las empresas objeto de estudio, el servicio al cliente está enfocado en el alcance de la satisfacción al cliente.

En lo concerniente a la gestión de inventario, los encuestados señalaron que este resultado se puede interpretar que no todas las empresas objeto de estudio analizan los costos de aprovisionamiento del stock de inventario. Cabe destacar que esta situación pone a dichas empresas en desventaja competitiva, donde las empresas deben garantizar un nivel deseado de disponibilidad de producto.

Para el almacenamiento los encuestados manifestaron que este indicador les permite a sus empresas, controlar eficientemente el flujo de materiales, del mismo modo la distribución abarca el conjunto de operaciones llevadas a cabo para que el producto recorra el camino que dista desde su punto de producción hasta el consumo.
Con respecto al indicador compras, se conoció que las empresas siempre realizan la gestión de compras en función de obtener bajos costos con alta calidad de los productos adquiridos, evidenciado una muy alta presencia del indicador; finalmente con respecto al indicador sistemas de información se conoció que en dichas empresas siempre los sistemas de información de gestión logística brindan apoyo a la toma de decisiones, logrando ventajas competitivas a través de su implantación.

Finalmente, las empresas petroquímicas de la Costa Oriental del Lago, deben prepararse tomando en cuenta el contexto interno, así como externos llenos de dificultades, pero también, de oportunidades y retos, los cuales deben enfrentar a través de sus fortalezas, para orientar desde una postura científica, las transformaciones que se requieren en el ámbito que abarca la gestión logística en sus procesos operacionales.

\section{REFERENCIAS}

Anaya, J. (2014). Almacenes: análisis y diseño organizacional. España: Editorial ESIC.

Arias, F. (2012). Fundamentos básicos de la investigación científica. Caracas. Venezuela: Universidad Central de Venezuela

Ballou, R. (2014). Gestión Logística. Administración de la cadena de suministro. México: Editorial Pearson-Prentice Hall

Bowersox, D, Closs, D y Cooper, B. (2007). Supply chain logistics management. Boston. EEUU: Editorial Mc Graw Hill

Calderón, E. (2002). Diseño y Layout de almacenes y centros de distribución. Madrid. España: Editorial Pirámide 
Carranza, O y Sanabria L. (2005). Logística: mejores prácticas en Latinoamérica. México: Editorial Tompson

Casanova, A; y Cuatrecasas, LI. (2012). Logística empresarial. Barcelona. España: Ediciones Gestión 2000 S.A

Coronado, J. (2008). Plan de mejoramiento de los procesos de compra y administración del almacén. Editorial ECOE. Bogotá. Colombia

Cruz, A.(2018). Gestión de inventarios. Barcelona. España: Ic Editorial

Hernández, R. Fernández, C. y Baptista, P. (2014). Metodología de la Investigación. México: Editorial Mc. Graw Hill

Laudon, Kenneth y Laudon, Jane. (2012). Sistemas de información gerencial. México DF, México: Pearson Educación

López, J. (2005). Gestión logística para la competitividad empresarial Caracas. Venezuela: Editorial Vanegas

López, R. (2014). Operaciones de almacenaje. Madrid. España: Editorial Paraninfo

Martínez, M y Brenzini, D. (2015). Sistemas de información en empresas de servicios petroleros de la Costa Oriental del Lago. Revista Ethos Venezolana. Vol. 7 (1), EneroJunio 2015, 58- 69

Mercado, A. (2006). El proceso de compra como parte elemental en la gestión logística del almacén. Editorial Caracol. Caracas. Venezuela

Mora, K. (2011). Medición de la gestión en la productividad y valor agregado. Editorial Libertadores. Bogotá. Colombia

Pulido, U. (2009). El proceso de almacenamiento como elemento básico de la logística de distribución e inventarios: eficacia y rentabilidad. Editorial Saturno. Bogotá, Colombia

Salas, J. (2017). Aportes de la gestión de inventarios. Editorial Uninorte. Universidad del Norte. Barranquilla. Colombia

Sánchez, O; y Méndez, A. (2006). Las actividades de gestión logística. Editorial Sypal. Caracas. Venezuela

Serna, H. (2006). Servicio al cliente: métodos de auditoria y medición. Bogotá. Colombia: Editorial Palacios y Cuestas 